\title{
Percepción sobre la competencia digital aplicada en estudiantes de la carrera de Medicina Veterinaria de la Universidad Católica de Santiago de Guayaquil ${ }^{*}$
}

Perception of the digital competence applied in students of the Veterinary Medicine career of the Catholic University

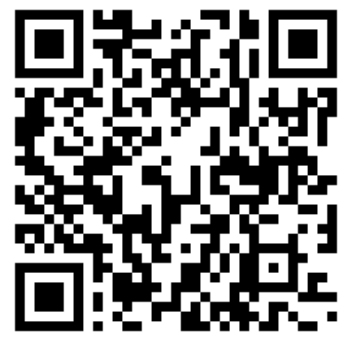
of Santiago de Guayaquil

Carlos G Manzo*

Fabiola de F Chonillo***

Lucila M Sylva $\mathrm{M}^{* * * *}$

\section{Resumen}

La atención y motivación en el aula como fuera de ella, por parte de los estudiantes universitarios dependen de los recursos didácticos que el docente implementa, aprovechando la red y sus posibilidades. El propósito de esta investigación cualitativa radica en evaluar la percepción de los estudiantes sobre el uso de la tecnología digital como aliado en el aula, incorporando elementos al entorno educativo como Pizarras interactivas, aulas virtuales $\mathrm{y}$ otros recursos electrónicos disponibles en la actualidad. Entre los materiales utilizados en esta investigación se consideró los resultados de

\footnotetext{
* Artículo original derivado del Proyecto "Competencias estudiantiles" Financiado por la Universidad Catolica Santiago de Guayaquil entre junio 2018 y mayo 2019

** Docente Universidad Católica Santiago de Guayaquil. Guayaquil, Ecuador. E-mail: carlos.manzo02@cu.ucsg.edu.ec. ORCID: http://orcid.org/0000-0003-1188-9581.

${ }^{* * *}$ Docente Universidad Católica Santiago de Guayaquil. Guayaquil, Ecuador. E-mail: fabiola.chonillo@cu.ucsg.edu.ec. ORCID: http://orcid.org/0000-0002-9844-2610.

**** Docente Universidad Católica Santiago de Guayaquil. Guayaquil, Ecuador. E-mail: lusylvamor@yahoo.com. ORCID: http://orcid.org/0000-0002-0928-3516.
}

\author{
Sinergias educativas \\ Enero - junio Vol. 5 -1 - 2020 \\ http://sinergiaseducativas.mx/index.php/revista/ \\ eISSN: 2662-6661 \\ revistasinergia@soyuo.mx \\ Recepción: 04 Septiembre 2018 \\ Aprobación: 16 Abril 2019 \\ Pag 29-45
}

\section{Disponible en}

http://www.redalyc.org/articulo.oa?id=57356143200 3

Atribución/Reconocimiento-NoComercialCompartirlgual 4.0 Licencia Pública Internacional - CC BY-NC-SA 4.0 https://creativecommons.org/licenses/by-ncsa/4.0/legalcode.es 
encuestas dirigidas a estudiantes sobre la percepción de la competencia digital en la enseñanza aprendizaje donde se evaluaron 7 preguntas importantes sobre la utilización de la tecnología como herramienta de mejora de la enseñanza-aprendizaje. Los estudiantes en su mayoría calificaron muy buena el uso de estas herramientas para la práctica docente tanto en escenarios en aula, así como en laboratorios o escenarios reales, lo que coincide con varios autores sobre la eficacia de la tecnología digital aplicada en clases.

Palabras clave: innovación, tecnología, digital, percepción.

\section{Abstract}

The attention and motivation in the classroom as outside of it, by university students depend on the teaching resources that the teacher implements, taking advantage of the network and its possibilities. The purpose of this qualitative research is to evaluate the students' perception of the use of digital technology as an ally in the classroom, incorporating elements into the educational environment such as interactive whiteboards, virtual classrooms and other electronic resources available today. Among the materials used in this research were the results of surveys aimed at students on the perception of digital competence in teaching learning where 7 important questions about the use of technology were evaluated as a tool for improving teaching-learning. The students mostly rated the use of these tools for teaching practice both in classroom settings, as well as in laboratories or real settings, which coincides with several authors on the effectiveness of digital technology applied in classes. Key words: innovation, technology, digital, perception.

\section{Introducción}

Según señala Ramsden, (2007) en la enseñanza académica, es necesario contar con herramientas necesarias para conseguir la atención y mejorar el aprendizaje en los estudiantes, se espera que el docente, diseñe cursos y aplique métodos de enseñanza adecuados para cumplir con los requerimientos de una población estudiantil heterogénea, que sepa lidiar con grupos numerosos de estudiantes, que utilice apropiadamente las nuevas tecnologías de la información 
y la comunicación pero ésta va de la mano de las técnicas empleadas para ello, se requieren herramientas denominadas competencias.

(Cacheiro, Medina, Domínguez 2019; Domínguez, Leví, Ramos, Sánchez, 2013). han evidenciado que la plataforma y su principal instrumento de comunicación: el foro, promueve una activa colaboración entre docente y discentes, a la vez que demanda una intensa actividad ante las necesidades de un mundo globalizado, y de encuentro entre culturas, también afirman que los instrumentos aplicados nos permiten evaluar la percepción de los estudiantes encuestados, con respecto a la competencia digital. Para el presente estudio se requirió los siguientes materiales: filmadora, laboratorio, computadora, software aulas, libreta de apuntes, bolígrafo; se realizó una tabla en Excel donde se clasificó los datos de cada estudiante evaluado, colocando el resultado sobre la encuesta de la competencia en estudio. La competencia digital en la función docente se concreta en una guía didáctica innovadora y el plan de formación que cada docente ha de reelaborar, precisar y trabajar con la mayor autonomía, mediante la incorporación de recursos como la plataforma.

El objetivo de ésta investigación pretende visualizar cómo los involucrados perciben la metodología de enseñanza sobre la competencia digital impartida por el docente. Entre las competencias ejecutadas durante el curso por los docentes mencionamos: competencia de comunicación, de innovación, digital, de investigación. Cada una de estas competencias responde a un solo objetivo, mejora del escenario enseñanza aprendizaje valiéndose de la búsqueda de nuevas tendencias como desafíos en la Educación Superior.

Castañeda, Carrillo, \& Zaira, (2013) afirman que. "Nos encontramos en pleno siglo XXI, ya la globalización, las nuevas tecnologías para la comunicación y la información (TIC), la era digital, el Internet, y ahora Internet 2.0 son palabras y hechos demasiado cotidianos".

Ramírez (2001), Afirma, en una investigación comparativa, con cuatro países latinoamericanos acerca del uso de las tecnologías de información y comunicación, encontró que, en los años recientes, los trabajos de investigación presentados en eventos internacionales sobre TIC y educación se ha incrementado de manera considerable. 
Según la expectativa se prevé que, con el uso de estas competencias, el alumnado dispondría de nuevas herramientas que le sirvan para la comprensión, asimilación y desarrollo de habilidades y destrezas necesarias para su profesión.

Según Baelo y Cantón (2009) entre las ventajas del uso de los diferentes medios tecnológicos tales podríamos mencionar que, los contenidos se asimilan más rápido, desarrolla el pensamiento crítico, motiva, promueve el autoaprendizaje, lo que provoca trabajo en equipo. Genera autonomía, reutilización de los contenidos temáticos rápidamente, interacción entre los alumnos, facilita la comprensión, adquiere la habilidad de seleccionar la información. Facilita la labor docente, siendo su aplicación enorme. Es visual y auditiva, puede ser Presencial o Representativa, existe un aprendizaje oculto, es decir se aprenden tópicos aparte del tema principal. Es más accesible desde el punto de vista económico y rápido, permitiendo transferir grandes cantidades de información más apegada a la realidad y contactar con expertos en la materia, e intercambiar opiniones con gente de otros países o regiones con culturas muy diferentes a la local.

Entre sus desventajas se puede mencionar que," Si no se tiene sustento pedagógico, puede frenar el aprendizaje. Elimina la posibilidad de desarrollar habilidades físicas. Si hay fallas en los equipos provoca frustración, además que con la falla de energía eléctrica es necesario cambiar de estrategia. Las tecnologías suelen estar sólo al alcance de algunos sectores de la sociedad. Si el autoaprendizaje se realiza en forma eficiente, deja a un lado al docente. Si el facilitador o docente no está preparado para utilizarla se excluye. Si el aprendizaje se da en línea, carece de calor humano. No hay interacción entre docente-discente o al menos disminuye. Las labores se dejan para el último momento. Se depende del equipo. Si no se cuenta con tecnología móvil, se limita el avance. Si no se cuenta con una disciplina adecuada genera malos hábitos. La evaluación tiende a no ser honesta. Si no se eligen sitios o portales adecuados podemos caer en el engaño o lo que es peor caer en el no aprender o hacernos de conocimientos falsos" Tecnología Educativa - UAMCEH (2010).

Arreola (2016) menciona que "El docente requiere ampliar su abanico de posibilidades para presentar los contenidos con procesos 
y ambientes innovadores y atractivos, versátiles y vigentes al momento socio histórico en el cual se desenvuelven, esto como respuesta a las características de los estudiantes actuales, quienes viven en la inmediatez y la multireferencialidad, ya que el uso de tecnologías de la información provee de herramientas de fácil acceso y pronta respuesta".

Según Murillo y Hernández (2010), citados en Arreola, (2017) expone cuatro factores impactan en el sentido motivacional que el docente ha de promover en los estudiantes al planear y desarrollar los contenidos de aprendizaje en el aula".

La opción por un modelo de competencias digitales a partir de las funciones y roles deviene de nuestro propio planteamiento escenario profesional (contexto) y las funciones que son de su incumbencia en el mismo (Pozos, 2011, 2013; Tejada, 2009).

Álvarez (2016) Afirma "al igual que los futuros docentes deben ser competentes en diversas áreas, también deben incorporar en su formación, el aprendizaje y actualización en procesos de enseñanzaaprendizaje mediados por las TIC, ya que así serán capaces de guiar y enseñar al alumnado para que mejore su competencia digital".

El profesor debe ser competente en instrumentar el ambiente digital empleando la información y el conocimiento de otros pares, colaborando en la innovación de materiales didácticos y colaborar a la democratización del conocimiento. Por tanto, el maestro precisa perfeccionar competencias digitales en el manejo de estos recursos (Aguirre \& Ruiz, 2012).

Según lo explican Ruíz, Márquez \& Álvarez (2018) las competencias que deben ostentarse para realizar una práctica docente son aquellas que construyen una cultura socioeducativa comenzando por: la empatía con sus estudiantes 97 digitales, liderazgo interactivo, vincular los conocimientos educativos con la praxis del entorno real y desde luego promover una evaluación de las competencias digitales centrándose en el aprendizaje profundo, y estas con lo referente a las competencias digitales sobre el aprendizaje. 
A este respecto, en el informe de Bioeduca (2006), se señalaba la existencia en España de unos 350.000 alumnos que llevaban a cabo alguna actividad formativa a través de la red y de éstos, un $50 \%$ son universitarios. En el informe se indica que uno de cada diez universitarios españoles ya es virtual, no acude a las aulas tradicionales, y dicha tendencia se está acrecentando, de tal manera que se estima que antes de finalizar el 2010, en España, un 10\% del alumnado total universitario desarrollará sus estudios de forma virtual o semipresencial.

Las competencias informacionales son importantes en el saber y hacer del docente como por ejemplo en el uso de datos para la nube, redes sociales para intercambio de información, y publicación de contenidos de la web 2.0. Si se considera que el conocimiento está en la red entonces el docente es quien deberá acompañar a los estudiantes digitales en su proceso de aprendizaje ya que los recursos digitales no guían por si solo es por eso por lo que la labor docente es tan importante como las instituciones educativas (Viñals, \& Cuenca, 2016).

\section{Materiales y métodos}

En este proyecto se aplicó un enfoque metodológico de tipo cuanticualitativo y de diseño no experimental, contrastando los datos mediante estudios descriptivos y correlacionales. Los encuestados fueron estudiantes de la Carrera de Medicina Veterinaria de la UCSG, quienes aportaron con sus criterios en el desarrollo de este estudio. Concordando con la investigación de (Pérez-Navío, Medina \& Cachón-Zagalaz, 2019; Medina et al., 2013), indican que la metodología integra la visión cuanti-cualitativa a partir de un cuestionario previo que se ha aplicado en varias investigaciones procediéndose en este estudio a optimizar la validez y fiabilidad, adaptándolo a esta nueva realidad universitaria, singularmente las preguntas abiertas, y profundizando en el análisis de los textos emergidos de los grupos de discusión.

Se consideró para este estudio a estudiantes de la carrera de medicina veterinaria, con quienes se trabajó para el desarrollo de esta investigación. La herramienta competencia digital se utilizó para la mejora aprendizaje colaborativo, además permiten al docente 
innovar y dinamizar el proceso de enseñanza aprendizaje de una forma asertiva. La muestra está constituida por 25 estudiantes, comprendidos entre 19 a 22 años, Para diseñar el cuestionario se procedió con la elaboración y selección de preguntas, a partir de una revisión de los instrumentos ya creados para este fin, adaptando el cuestionario a la realidad actual (Cabero, Llorente \& Marín, 2010)

Tomando como referencia al trabajo de Cabero, Llorente y Marín, (2006); Guzmán, (2008); Prendes, (2010), se procedió con la introducción de las preguntas con la colaboración de docentes e investigadores del ámbito de la metodología de la investigación, la didáctica y las TIC. Seguidamente se analizaron las aportaciones de los expertos y se realizaron los cambios propuestos.

Según Fernández García, A., García Llamas, J. L., \& Pérez Serrano, G. (2014) se ha recurrido a una metodología mixta. Su riqueza combina los métodos cuantitativos y cualitativos permitiéndonos obtener información y contraste desde diferentes perspectivas. La finalidad es verificar los resultados de la investigación.

La validación del cuestionario se realizó mediante docentes de la carrera implicando al profesorado e investigadores en la comprensión y transformación de la clase, analizando la citada competencia digital y su impacto en los estudiantes, El análisis de datos se ejecutó utilizando tablas en Excel en la que se evaluaron 7 preguntas importantes sobre la utilización de la tecnología como herramienta de mejora de la enseñanza-aprendizaje educativo.

\section{Resultados}

Los resultados obtenidos a través de esta encuesta y que se realizó a los estudiantes de la Carrera de Medicina Veterinaria, quienes participaron durante los tres semestres del Proyecto de Investigación de la Universidad Católica de Santiago de Guayaquil y la Universidad Nacional de España UNED.es, fueron los siguientes:

En la Tabla 1, podemos observar la evaluación realizada por los estudiantes a la metodología empleada por el docente como Competencia digital, en base a preguntas que fueron calificadas con 
una escala del 1 al 5 el nivel de satisfacción (NS), donde 1 indica nada satisfactorio, hasta llegar al 5 que indica muy satisfactorio.

Tabla 1. Análisis de las Competencias digitales

\begin{tabular}{|c|c|c|c|c|}
\hline $\begin{array}{l}\text { COMPETENCIA } \\
\text { DIGITAL }\end{array}$ & 1 & 2 & 4 & 5 \\
\hline $\begin{array}{l}\text { El docente relaciona } \\
\text { la compe-tencia } \\
\text { digital con su } \\
\text { historial profesional } \\
\text { innovadora }\end{array}$ & 0 & ) & 8 & 12 \\
\hline $\begin{array}{lr}\text { La práctica } & \text { del } \\
\text { proceso } & \text { de } \\
\text { enseñanza } & - \\
\text { aprendizaje } & \text { se } \\
\text { asume como un } \\
\text { proyecto de mejora } \\
\text { permanente }\end{array}$ & 0 & ) & 9 & 11 \\
\hline $\begin{array}{l}\text { Genera actividades } \\
\text { educativas } \\
\text { coherentes con el } \\
\text { modelo didáctico } \\
\text { elegido }\end{array}$ & 0 & ) & 10 & 12 \\
\hline
\end{tabular}

El profesor presenta ideas creativas a los $\begin{array}{llllll}0 & 1 & 2 & 8 & 14\end{array}$ estudiantes

\begin{tabular}{|c|c|c|}
\hline $\begin{array}{lrr}\text { Diseña } & \text { tareas } \\
\text { originales } & & \text { que } \\
\text { motivan a } & \text { los } \\
\text { estudiantes } & & \end{array}$ & $\begin{array}{llll}0 & 2 & 3 & 7\end{array}$ & 13 \\
\hline $\begin{array}{l}\text { Ofrece a los } \\
\text { estudiantes méto- } \\
\text { dos innovadores que } \\
\text { facilitan la actitud } \\
\text { investigadora }\end{array}$ & $\begin{array}{llll}0 & 2 & 2 & 10\end{array}$ & 11 \\
\hline
\end{tabular}




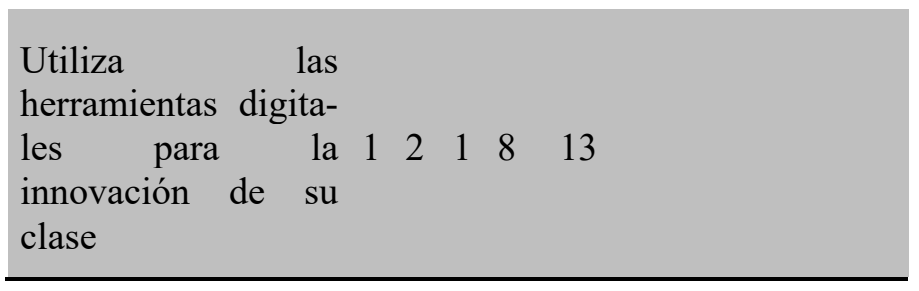

Evaluación de las competencias digitales del docente.

Las tecnologías aplicadas a la docencia permiten al docente identificarse con los estudiantes, ya que ellos viven en esta era donde el uso de las tecnologías predomina en todos los ámbitos. Esta competencia del docente es considerada por el $48 \%$ de los encuestados como muy satisfactoria. Para el $32 \%$ la consideró como satisfactoria; pero para un $20 \%$ fue catalogada como medianamente satisfactoria.

Las herramientas digitales permiten interactuar al estudiante con el conocimiento, aprendiendo de manera simultánea con la dinámica que ofrecen las tecnologías, logrando la mejora continua esperada en la educación. Esta competencia fue considerada por el $44 \%$ de los encuestados como muy satisfactoria, mientras que, para el $36 \%$ fue satisfactoria; para el $20 \%$ restante esta competencia digital fue catalogada como medianamente satisfactoria.

Los modelos didácticos empleados en las clases deben ser coherentes con la temática, con el nivel de enseñanza para que pueda cumplir su objetivo. Esta competencia fue considerada como muy satisfactoria por el $48 \%$ de los encuestados. El $40 \%$ de los estudiantes lo catalogaron como satisfactorio; mientras que para el restante $12 \%$, esta competencia fue considerada como medianamente satisfactoria.

La creatividad aplicada a la enseñanza permite diseñar o crear diferentes formas de llegar al estudiante para lograr que se involucre en su aprendizaje. Este componente del docente fue considerado como muy satisfactorio para el $56 \%$ de los encuestados; para el 32 $\%$ lo catalogaron como satisfactorio; medianamente satisfactorio fue considerada por el $8 \%$, mientras que, para un estudiante $(4 \%)$ este componente es poco satisfactorio.

Las tareas enviadas, que aplican la tecnología, se emplean como una herramienta para afianzar los conceptos, incentivar la investigación 
y ampliar los conocimientos impartidos en clase, permitiendo al estudiante adquirir argumentos con los cuales participar en los foros y demás actividades académicas. Esta competencia del docente fue considerada como muy satisfactoria por el $52 \%$ de los encuestados; para el $28 \%$ fue catalogada como satisfactoria; medianamente satisfactoria fue evaluada por el $12 \%$, mientras que para el $8 \%$ de los estudiantes fue considerada como poco satisfactoria.

El uso de la tecnología en la enseñanza, permite acercarse a los avances y beneficios que esta conlleva en los estudiantes, relacionando la investigación como el pilar fundamental para adquirir el conocimiento necesario para ejercer la profesión con seguridad. Esta competencia del docente fue considerada muy satisfactoria por el $44 \%$ de los encuestados; satisfactoria fue para el $40 \%$; siendo medianamente satisfactoria y poco satisfactoria para un $8 \%$ de los estudiantes, respectivamente.

El uso de las herramientas digitales por parte del docente, permite difundir los conocimientos de manera dinámica, comprensiva y más atractiva por parte de los estudiantes. Esta competencia fue calificada como muy satisfactoria por el $52 \%$ de los encuestados; satisfactorio fue para el $32 \%$; medianamente satisfactoria para el 4 $\%$; poco satisfactorio para el $8 \% \mathrm{y}$, finalmente para un $4 \%$ les resultó nada satisfactorio.

\section{Discusión}

La ejecución de este trabajo concuerda con Cabero (2013), Medina et al., (2013) y Cacheiro et al. (2018) con la investigación realizada por la competencia digital presentan elementos coincidentes, subrayándose que el desarrollo de la competencia digital, es entendido como un nuevo modo de reelaborar, precisar y trabajar con autonomía el uso creativo de los recursos didácticos en diferentes ámbitos, coincidiendo el reconocimiento de esta competencia con los trabajos de Ashwin et al. (2016).

También coincide con Lucas-Molina (2014), quienes afirman que el trabajo colaborativo en Redes, las prácticas reflexivas, actividades que propician una buena interacción en el aula y sobre todo la formación permanente. 
Aunque según UAMCEH (2010). existen marcadas desventajas sobre el uso de la tecnología digital en el aula por la facilidad con que éste medio de aprendizaje se desvíe del objetivo principal por el acceso a plataformas y páginas de contenido prohibido, así como a la dependencia directa de La tecnología como medio de vida, y la poca comunicación en el entorno familiar, fraudes, aumento de plagio, más desempleo, exceso de consumismo, genera vicios, hace que los estudiantes cada vez se esfuercen menos por realizar sus tareas, entre otras.

\section{Conclusiones}

En este estudio, se demuestra que los estudiantes son conscientes de la utilidad de estas herramientas para su futuro profesional, para la práctica docente tanto en escenarios en aula, así como en laboratorios o escenarios reales, en los datos obtenidos se valora la predisposición docente para aplicar estas herramientas de mejora del aprendizaje, como uno de los puntos más valorados en este estudio se menciona las grabaciones de mini videos, que se realizaron individual, luego en grupo.

En conclusión, todo depende de cómo sea usada. Si es mal manejada el resultado puede ser desastroso, pero si se enfoca de manera correcta puede ser una excelente herramienta de aprendizaje. Definitivamente abre muchas puertas muy útiles y otras que puede que no sean tan útiles. Es importante el continuo seguimiento a las tareas implantadas en el aula para redirigir eficazmente al estudiantado.

\section{Referencias}

Aguirre, G. \& Ruiz, M. (2012). Competencias digitales y docencia: una experiencia desde la práctica universitaria. Innovación Educativa. Recuperado http://www.scielo.org.mx/pdf/ie/v12n59/v12n59a9.pdf

de:

Álvarez, J. F. (2016). La Formación en TIC del profesorado de Secundaria del Estado Español. Un análisis desde la percepción docente. Revista de Cie. Formación docente en tics. ¿están los docentes preparados para la (r)evolución tic? Número 1 Vol 4 Recuperado de: https://www.redalyc.org/pdf/3498/349832327003.pdf 
Arreola, R. (2017) La transformación de la práctica docente: Editorial Universitaria

Arreola, R., Montiel, S. \& Calvo, A. (2016) Artículo de Investigación: La transformación de la práctica docente: competencias docentes y habilidades cognitivas Año 4 Número 1 Pag.12 Recuperado de: http://cresur.edu.mx/OJS/index.php/CRESUR_REIIE/article/view/ $310 / 228$

Ashwin, P., Abbas, A., \& McLean, M. (2016). Revista: Teaching in Higher Education: The influence of curricula content on English sociology students' transformations: the case of feminist knowledge 42(6), 962-977. Recuperado de: https://srhe.tandfonline.com/doi/full/10.1080/13562517.2016.1155 551

Baelo R., \& Cantón I. (2009). Las Tecnologías de la información y la comunicación en la educación superior. Estudio descriptivo y de revisión. Revista Iberioamericasna de Educación, 50(7), 1-12. Recuperado de: https://rua.ua.es/dspace/bitstream/10045/35701/1/Tesis_Laguna_Se govia.pdf

BIOEDUCA (2006). Informe Bioeduca 2006. La educación a distancia. Madrid: Observatorio Español de Internet. Recuperado de: https://www.researchgate.net/publication/38291654_Las_tecnologi as_de_la_informacion_y_la_comunicacion_en_la_educacion_super ior_Estudio_descriptivo_y_de_revision

Cabero, J. \& Llorente, M. (Dirs.) (2006). La rosa de los vientos: Dominios tecnológicos de las TIC por los estudiantes. Sevilla: Grupo de Investigación Didáctica. Recuperado de: https://www.redalyc.org/pdf/368/36841180002.pdf

Cabero, J., Llorente, M. \& Marín, D. (2010). Hacia el diseño de un instrumento diagnóstico de «competencias tecnológicas del profesorado» universitario. Revista Iberoamericana de Educación, 52 (7), $1-12 \quad$ Recuperado de: https://www.redalyc.org/pdf/368/36841180002.pdf

Cabero, J. y Barroso, J. (2013). La formación virtual en el nuevo entramado 2.0 el e-learning 2.0 En I. Aguaded, y J. Cabero, (Cood). Tecnologías y medios para la educación en la sociedad. (pp.12-23). 


\section{Madrid:}

Alianza.

Recuperado

de: https://www.redalyc.org/pdf/706/70629509005.pdf

Cacheiro, Medina, Domínguez \& Medina (2019). The learning platform in distance higher education: student's perceptions. Turkish Online Journal of Distance Education, TOJDE, 20(1), 71-95. Recuperado

de: https://www.researchgate.net/publication/320981803_Las_compete ncias_docentes_diagnostico_y_actividades_innovadoras_para_su_d esarrollo_en_un_modelo_de_educacion_a_distancia/link/5a $059 \mathrm{e} \overline{\mathrm{d}} \mathrm{d}$ a6fdcceda 0348 dee/download

Castañeda, A., Carrillo, J., \& Zaira., Q. (2013). El uso de las TIC en Educación Primaria: la Experiencia ENCICLOMEDIA. Durango, Dgo: Red de Investigadores Educativos, A.C (pág. 14). https://docplayer.es/5647309-El-uso-de-las-tic-en-educacionprimaria-la-experiencia-enciclomedia.html

Estados Unidos Mexicanos. Secretaria de Educación Pública (2016). Planeación integral de la Educación Superior. Ciudad de México. http://www.pides.mx/pides_2017/

Fernández García, A., García Llamas, J. L., \& Pérez Serrano, G. (2014). Los Programas Universitarios de Mayores y su contribución al aprendizaje a lo largo de la vida. Revista Complutense De Educación, $\quad 25 \quad$ (2), 521-540. https://doi.org/10.5209/rev_RCED.2014.v25.n2.42058

Guzmán, T. (2008). Las tecnologías de la información y la comunicación en la Universidad Autónoma de Querétaro: propuesta estratégica para su integración. Tesis doctoral. Universitat Rovira i Virgili. Recuperado de: https://www.redalyc.org/pdf/368/36841180002.pdf

Lucas-Molina, B. (2014). Competencias que configuran el perfil del docente de primaria. Análisis de la opinión del alumnado del grado de Educación primaria. Aula Abierta, 42, 53-60. Recuperado de: http://www.scielo.org.co/pdf/teclo/v21n41/v21n41a08.pdf

Medina, A., Domínguez, M.C., Leví, G., Ramos, E., Sánchez, C., Cacheiro, M.L. (2013). Formación del profesorado: Actividades innovadoras para el dominio de las competencias docentes. Madrid: Editorial Universitaria Ramon Areces. 1ra Edición 26-07-2013 Recuperado de: https://www.cerasa.es/libro/formacion-delprofesorado_50071/ 
Murillo, F. J., Krichesky, G., Castro, A. y Hernández, R. (2010). Liderazgo escolar para la Inclusión y la Justicia Social. Revista Latinoamericana de Educación Inclusiva, 4 (1), pp. 169-186. Recuperado de: https://www.redalyc.org/pdf/551/55129541007.pdf

Pérez, E., Medina, M.C. \& Cachón-Zagalaz, J. (2019). Perception of the Professional Competences of Last Year's Students of PrePrimary Education and Primary Education Degrees and Students of Training Teachers Mater. Journal New Approaches in Educational Research, 8(1), 58-65.58-65. Recuperado de: https://www.unioviedo.es/reunido/index.php/AA/article/view/1334 9

Prendes, M.P. (dir) (2010). Competencias TIC para la docencia en la Universidad Pública española. Indicadores y propuestas para la definición de buenas prácticas. Revista Digital de Educación en docencia universitaria Recuperado de: http://www.scielo.org.pe/pdf/ridu/v12n2/a04v12n2.pdf

Pozos, K. V. (2013). Digital competence of Higher Education Teachers for KnowledgeSociety: A Preliminary approach of a Need Assessment Study in Mexican Universities. ICERI2013 -6th International Conference of Education, Research and Innovation. International Association of Technology, Education and Development, Recuperado de: https://www.researchgate.net/publication/323535297_Nuevos_esce narios_y_competencias_digitales_docentes_Hacia_la_profesionaliz acion_docente_con_TIC

Ramírez, J. L. (2001). "Educación y computadoras: una aproximación al estado actual de su investigación en México", Revista Mexicana de Investigación Educativa, vol. VI, núm. 11, enero-abril, pp. 119-138. Recuperado de: http://www.comie.org.mx/documentos/rmie/v11/n28/pdf/rmiev11n 28scB02n03es.pdf

P. Ramsden (2007), Learning to Teach in Higher Education, Londres/Nueva York, Routledge Falmer. Recuperado de: http://www.scielo.org.mx/pdf/peredu/v33nspe/v33nspea12.pdf

Ruíz-Sepúlveda, D., Márquez-Rodríguez, A. \& Alvarez-Amezcua (2018). El profesor digital como influencer académico. Comunicar FCC; no.1 58-65. Recuperado de: http://www.cuadernosartesanos.org/2019/cac153.pdf 
Tejada, J. (2013). Artículo Científico: La formación de competencias profesionales a través del aprendizaje servicio. Cultura y educación, 25 (3) 285-294. Recuperado de: https://www.researchgate.net/publication/269517117_Formando_fo rmadores_nuevos_escenarios_y_competencias_digitales_docentes Training_trainers_new_scenarios_and_trainers'_digital_competenci es

Viñals Blanco, A., \& Cuenca Amigo, J. (2016). El rol del docente en la era digital. Revista Interuniversitaria de Formación del Profesorado, 30 (2), 103-114. Recuperado de: https://www.redalyc.org/pdf/274/27447325008.pdf 